\title{
Lymph node spread of gallbladder cancer from the perspective of embryologically-based anatomy and significance of the lymphatic basin along the embryonic right hepatic artery
}

\author{
HISATOSHI NAKAGAWARA, HIDEHIRO TAJIMA, TOMOHARU MIYASHITA, HIROHISA KITAGAWA, \\ ISAMU MAKINO, SEISHO SAKAI, HIRONORI HAYASHI, SHINICHI NAKANUMA, \\ KATSUNOBU OYAMA, HIROYUKI TAKAMURA, SACHIO FUSHIDA, \\ TAKASHI FUJIMURA and TETSUO OHTA
}

\begin{abstract}
Division of Cancer Medicine, Department of Gastroenterologic Surgery, Graduate School of Medicine Science, Kanazawa University, Kanazawa, Ishikawa 920-0934, Japan
\end{abstract}

Received June 4, 2014; Accepted July 3, 2014

DOI: $10.3892 / \mathrm{mco} .2014 .342$

\begin{abstract}
Lymph node metastasis from gallbladder cancer is often found in the pericholedochal area; however, these regional lymph nodes are not typically accompanied by arteries. We hypothesized that the artery accompanying pericholedochal lymph nodes was either the regressed embryonic right hepatic artery (eRHA) or an aberrant right hepatic artery (aRHA) remaining without regression. This study aimed to determine the artery supplying pericholedochal lymph nodes. We obtained serial tissue sections of resected specimens from 10 patients who underwent pancreaticoduodenectomy with combined resection of the superior mesenteric artery and vein and investigated the association between the distribution of enlarged lymph nodes and the course of blood vessels in each section. In 2 cases with aRHA, enlarged lymph nodes were distributed in the posterosuperior area, pancreaticoduodenal region and retroportal area along this artery. By contrast, no blood vessels accompanied enlarged lymph nodes in 8 patients exhibiting a normal hepatic artery branching pattern, although these nodes exhibited a distribution pattern similar to that of patients with the aRHA. Thus, the artery supplying pericholedochal lymph nodes appears to be either the regressed eRHA or an aRHA persisting without regression.
\end{abstract}

Correspondence to: $\mathrm{Dr}$ Hisatoshi Nakagawara, Division of Cancer Medicine, Department of Gastroenterologic Surgery, Graduate School of Medicine Science, Kanazawa University, 13-1 Takaramachi, Kanazawa, Ishikawa 920-8641, Japan

E-mail: nakagawarahisatoshi@gmail.com

Key words: right hepatic artery, embryonic right hepatic artery, lymph node metastasis, gallbladder cancer, lymphadenectomy, pericholedochal lymph node

\section{Introduction}

The prognosis of gallbladder cancer (GBC) with lymph node metastases is poor. The 5 -year survival rate following surgical resection has been reported to be $65 \%$ for patients with no lymph node metastases and $<30 \%$ for those with regional lymph node metastases (pN1) (1). In the pN1 group, lymph node metastases characteristically arise in the pericholedochal area or the posterosuperior pancreaticoduodenal region, rather than around the hepatic artery $(2,3)$. However, no feeding arteries are present around the pericholedochal lymph nodes and posterosuperior pancreaticoduodenal lymph nodes (PPLNs), although lymph nodes and lymphatic vessels generally exist along a feeding artery (4-7).

We previously performed pancreaticoduodenectomy (PD) for pancreatic cancer in patients with an aberrant right hepatic artery (aRHA). In such cases, PPLNs were distributed along the aRHA. This artery is considered to represent an embryonic right hepatic artery (eRHA) that has not regressed. We hypothesized that the artery responsible for PPLNs would be the eRHA. If this hypothesis is accurate, lymphadenectomy for GBC should be performed based on the course of the eRHA (8). The aim of this study was to determine the artery supplying the PPLNs.

\section{Materials and methods}

Patients. Between 1997 and 2005, a total of 58 patients underwent PD for invasive pancreatic ductal cancer in the Department of Gastroenterologic Surgery at Kanazawa University. Among these cases, radical PD with major vessel resection [superior mesenteric artery (SMA) and superior mesenteric vein (SMV) resection] was performed in 18 patients for complete clearance of the soft tissues around these arteries (9). This study was approved by the Ethics Committee at our institution and was conducted in accordance with the Declaration of Helsinki.

Specimens. The resected specimens were immediately fixed in $10 \%$ neutral-buffered formaldehyde solution, then 
A

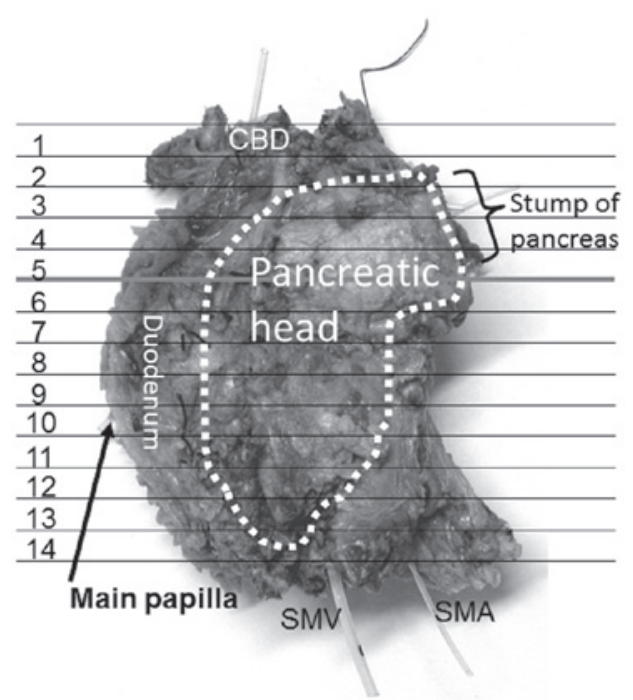

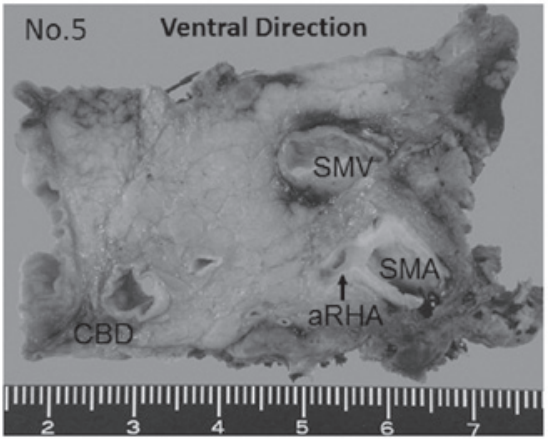

Figure 1. (A) Resected specimen of pancreaticoduodenectomy with combined resection of the superior mesenteric artery (SMA) and superior mesenteric vein (SMV). The resected specimens were cut horizontally into 5-mm tissue blocks corresponding to computed tomography images. Silicone catheters were inserted into the portal vein/SMV and the SMA. (B) One of the resected specimens, in which removal of the duodenum, pancreatic head, bile duct, SMV, SMA and surrounding connective tissue by en bloc resection allowed for the determination of the lymphatic route between the area surrounding the SMA and the pericholedochal area. CBD, common bile duct; aRHA, aberrant right hepatic artery.

A

B
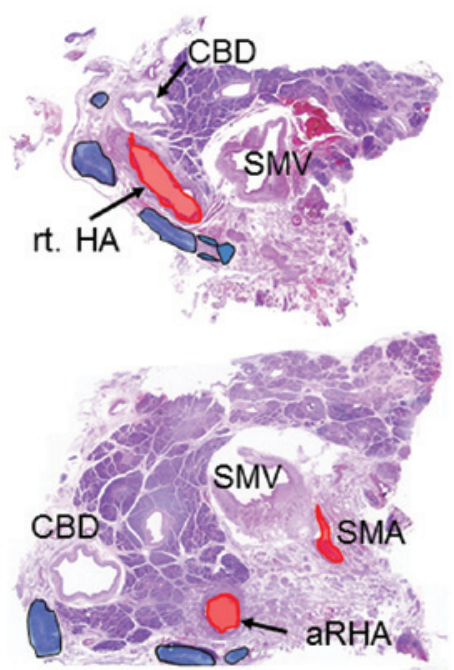

C

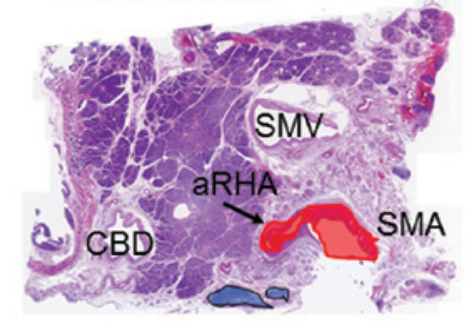

D

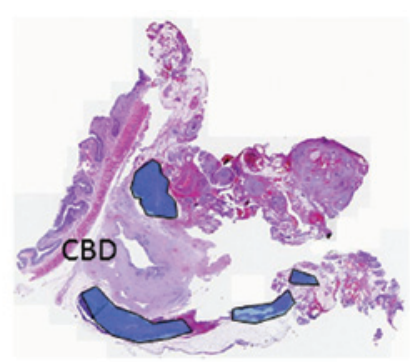

$\mathbf{E}$

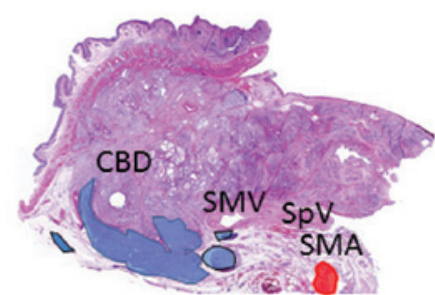

$\mathbf{F}$

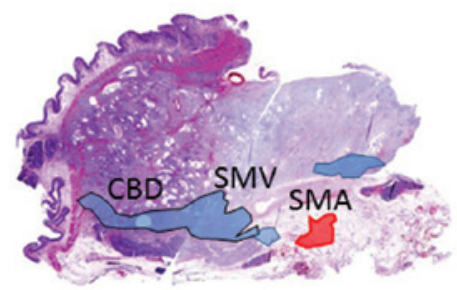

Figure 2. (A-C) Photographs of a resected specimen with an aberrant right hepatic artery (aRHA) [hematoxylin and eosin (H\&E) stain; original magnification, $x 1$ ]. (C) The aRHA branched from the right side of the superior mesenteric artery (SMA) and (B) crossed dorsal to the pancreatic head, then (A) proceeded to the right of the common bile duct (CBD). (A-C) The purple regions denote enlarged lymph nodes distributed along the aRHA. The red regions denote the arteries. (D-F) Photographs of a resected specimen with normal arterial branching (H\&E stain; original magnification, $\mathrm{x} 1$ ). (D-F) The distribution of lymph nodes resembles that in cases with an aRHA. SMV, superior mesenteric vein; rt. HA; right hepatic artery; SpV, splenic vein.

cut horizontally into 5-mm tissue blocks corresponding to computed tomography images, dehydrated and fixed in paraffin (Fig. 1). Finally, the specimens were cut into $5-\mu \mathrm{m}$ sections and stained with hematoxylin and eosin. Eight cases in which evaluation of the lymphatic route was difficult, due to failed fixation of specimens or development of pancreatic cancer lesions, were excluded from our study. For the evaluation, the patients were divided into 2 groups, those with an aRHA and 

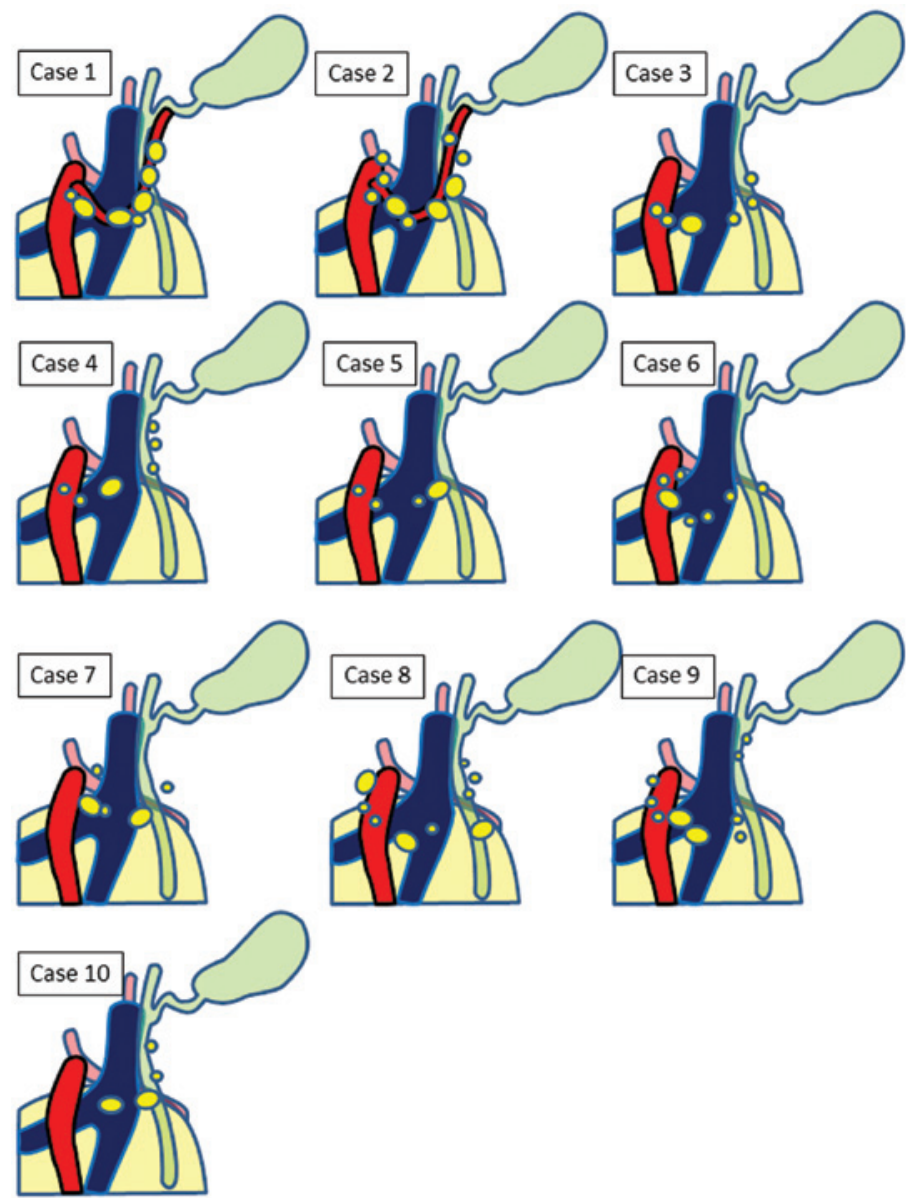

Figure 3. Schematic diagram of enlarged lymph nodes mapped to the resected specimens. The yellow regions represent enlarged lymph nodes. Cases 1 and 2 show an aberrant right hepatic artery. The remaining cases exhibit normal arterial branching.

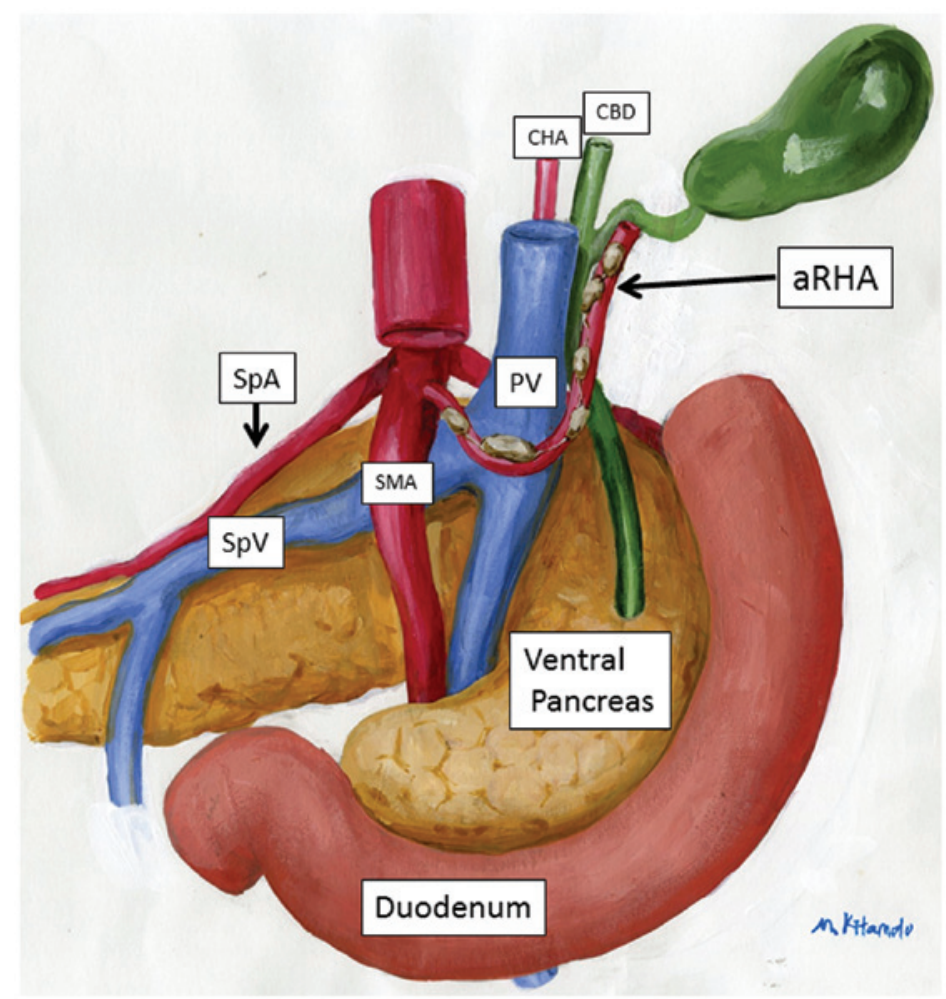

Figure 4. Schematic diagram of the distribution of lymph nodes from the gallbladder along the right aberrant hepatic artery (aRHA). Schematic diagram showing the course of lymph node distribution in the pericholedochal region dorsal to the portal vein (PV) along an aRHA. SMA, superior mesenteric artery; $\mathrm{SpA}$, splenic artery; $\mathrm{SpV}$, splenic vein; CHA, common hepatic artery; $\mathrm{CBD}$, common bile duct. 
those with a normal hepatic artery and no aRHA. Each section was carefully examined under a light microscope to determine the presence of lymph nodes around the blood vessels and their distribution (Fig. 2).

\section{Results}

Cases. Among the 18 cases, 10 met the inclusion criteria and were examined for the pattern of lymphatic spread. Among these, 2 patients had an aRHA and 8 exhibited normal arterial branching. Representative examples of cases with typical distribution of lymph nodes are shown in Fig. 2. In these specimens, removal of the duodenum, pancreatic head, bile duct, SMV, SMA and surrounding connective tissue by en bloc resection allowed for the determination of the lymphatic route between the area surrounding the SMA and the pericholedochal area (9).

Arterial pattern. Examination of the course of the arteries in the 2 cases with an aRHA revealed that the vessel branched from the right side of the SMA (Fig. 2C) and crossed dorsal to the SMV and pancreatic head (Fig. 2B), then proceeded to the right side of the bile duct (Fig. 2A). Observation of the lymph node distribution revealed enlarged PPLNs along the aRHA in both patients (Fig. 3). In the 8 patients with normal hepatic artery branching, enlarged lymph nodes were observed in the pericholedochal area, dorsal to the pancreatic head, in the retroportal area and on the right side of the SMA (Fig. 3). Interestingly, the lymph node distribution closely resembled that of cases with an aRHA, appearing with a similar distribution, but where a regressed eRHA would have passed (Fig. 4).

\section{Discussion}

Radical lymph node dissection for GBC is crucial (10) and understanding the pattern of lymph node metastatic spread from GBC is mandatory for surgeons $(11,12)$. This study demonstrated that PPLNs were distributed along the aRHA. In patients with a normal right hepatic artery, these lymph nodes were also distributed in a similar manner, suggesting distribution along the regressed eRHA.

During early human fetal life, 3 vessels exist, the embryonic left, middle and right hepatic arteries (13). During the developmental process, the embryonic left and right hepatic arteries regress and the middle hepatic artery persists. By adulthood, the embryonic middle hepatic artery has differentiated into the right, middle and left hepatic arteries. However, in some cases the eRHA persists without regression, representing an aRHA. The lymphatics travel primarily with blood vessels $(6,7)$. Even if the artery completely regresses, the accompanying lymphatic vessels, lymph nodes and nerves presumably remain (4). We therefore considered that the enlarged lymph nodes observed in cases with normal hepatic arterial branching were compatible with lymph nodes along a regressed eRHA.

An understanding of this concept may contribute to lymphadenectomy for GBC. As the standard surgical treatment in colorectal cancer, intestinal resection and regional lymphadenectomy, including feeding arteries, is considered essential $(13,14)$. In GBC up to stage IIa, the frequency of lymph node metastasis in the pericholedochal area has been reported to be higher compared to that of lymph node metastasis around the hepatic artery $(2,3)$. The lymphatic pathway of PPLNs has been called 'the right route of lymphatic drainage of the gallbladder' in Japan and has been considered as the main lymphatic route requiring resection in the treatment of $\operatorname{GBC}(12,15,16)$. From the present study, the right route is considered part of the route of the eRHA. To completely remove the lymphatic basin in the eRHA route, resection of the nodes in the pericholedochal area, posterosuperior pancreaticoduodenal area and retroportal area should also be performed (14). This consideration is undoubtedly a key reason as to why the addition of PD is associated with improved prognosis for patients with GBC (17-19). We hypothesize that PD allows en bloc resection of the lymphatic basin along the eRHA. However, performing the surgery with awareness of the fetal pathway of lymphatic vessels may enable surgery with appropriate lymphadenectomy, without PD, for GBC.

Clearly, this hypothesis requires confirmation by further embryological studies; however, we suggest that surgery with an awareness of lymph node metastasis along the eRHA may lead to improved prognosis for patients with GBC and we suggest that the feeding artery for PPLNs is either the regressed eRHA or an aRHA persisting without regression.

\section{References}

1. Miyakawa S, Ishihara S, Horiguchi A, Takada T, Miyazaki M and Nagakawa T: Biliary tract cancer treatment: 5,584 results from the Biliary Tract Cancer Statistics Registry from 1998 to 2004 in Japan. J Hepatobiliary Pancreat Surg 16: 1-7, 2009.

2. Tsukada K, Kurosaki I, Uchida K, et al: Lymph node spread from carcinoma of the gallbladder. Cancer 80: 661-667, 1997.

3. Nagakawa T, Kayahara M, Ikeda S, et al: Biliary tract cancer treatment: results from the Biliary Tract Cancer Statistics Registry in Japan. J Hepatobiliary Pancreat Surg 9: 569-575, 2002.

4. Yi SQ, Shimokawa T, Akita K, Ohta T, Kayahara M, Miwa K and Tanaka S: Anatomical study of the pancreas in the house musk shrew (Suncus murinus), with special reference to the blood supply and innervation. Anat Rec A Discov Mol Cell Evol Biol 273: 630-635, 2003.

5. Cha YR, Fujita M, Butler M, Isogai S, Kochhan E, Siekmann AF and Weinstein BM: Chemokine signaling directs trunk lymphatic network formation along the preexisting blood vasculature. Dev Cell 22: 824-836, 2012.

6. Geudens I,Herpers R,Hermans K, et al: Role of delta-like-4/Notch in the formation and wiring of the lymphatic network in zebrafish. Arterioscler Thromb Vasc Biol 30: 1695-1702, 2010.

7. Liao S, Padera TP and Jain RK: Notch leads lymphatics and links them to blood vessels. Arterioscler Thromb Vasc Biol 30: 1682-1683, 2010

8. Miwa K, Kinami S, Taniguchi K, Fushida S, Fujimura T and Nonomura A: Mapping sentinel nodes in patients with early-stage gastric carcinoma. Br J Surg 90: 178-182, 2003.

9. Kitagawa H, Ohta T, Makino I, et al: Carcinomas of the ventral and dorsal pancreas exhibit different patterns of lymphatic spread. Front Biosci 13: 2728-2735, 2008.

10. Wang JD, Liu YB, Quan ZW, Li SG, Wang XF and Shen J: Role of regional lymphadenectomy in different stage of gallbladder carcinoma. Hepatogastroenterology 56: 593-596, 2009.

11. Uesaka K, Yasui K, Morimoto T, et al: Visualization of routes of lymphatic drainage of the gallbladder with a carbon particle suspension. J Am Coll Surg 183: 345-350, 1996.

12. Shirai Y, Yoshida K, Tsukada K, Ohtani T and Muto T: Identification of the regional lymphatic system of the gallbladder by vital staining. Br J Surg 79: 659-662, 1992.

13. Chen SL and Bilchik AJ: Resecting lymph nodes in colon cancer: more than a staging operation? Ann Surg Oncol 14: 2175-2176, 2007.

14. Takeuchi H and Kitagawa Y: Sentinel node navigation surgery in patients with early gastric cancer. Dig Surg 30: 104-111, 2013. 
15. Ito M, Mishima $Y$ and Sato T: An anatomical study of the lymphatic drainage of the gallbladder. Surg Radiol Anat 13: 89-104, 1991.

16. Deki H and Sato T: An anatomic study of the peripancreatic lymphatics. Surg Radiol Anat 10: 121-135, 1988.

17. Shirai Y, Ohtani T, Tsukada $K$ and Hatakeyama $K$ : Pancreaticoduodenectomy for gallbladder cancer with peripancreatic nodal metastases. Hepatogastroenterology 44: 376-377, 1997.
18. Doty JR, Cameron JL, Yeo CJ, Campbell K, Coleman J and Hruban RH: Cholecystectomy, liver resection, and pylorus-preserving pancreaticoduodenectomy for gallbladder cancer: report of five cases. J Gastrointest Surg 6: 776-780, 2002.

19. Shirai Y, Wakai T and Hatakeyama K: Radical lymph node dissection for gallbladder cancer: indications and limitations. Surg Oncol Clin N Am 16: 221-232, 2007. 